

\title{
Inteligencia artificial en otorrinolaringología
}

En 1997 Garry Kasparov -campeón mundial de ajedrez-fue derrotado por Deep Blue, un super computador desarrollado especialmente para este evento por la empresa IBM. Si bien esta confrontación marcó un hito en la historia de la computación, la estrategia de procesamiento utilizada por Deep Blue fue de fuerza bruta, basada en una gran capacidad de almacenamiento y procesamiento de datos, permitiendo al computador analizar todas las jugadas posibles sin un criterio que involucre aprendizaje, decisión o jerarquía.

Ya han pasado más de 20 años de este acontecimiento, y la tecnología disponible ha avanzado a un paso frenético, de manera que contamos con computadores muy poderosos a relativamente bajos costos, y la inteligencia artificial cuenta con estrategias de procesamiento que involucran aprendizaje de máquinas (machine learning) en redes neurales artificiales profundas o deep learning, muy superiores a la estrategia de fuerza bruta que utilizaba Deep Blue.

En la actualidad, la inteligencia artificial puede ser aplicada en diversas áreas de la medicina, incluyendo el diagnóstico clínico, la cirugía robótica, dispositivos de ayuda médica 0 el desarrollo de programas para mejorar la eficiencia del manejo de datos en redes hospitalarias. Algunos ejemplos en que la inteligencia artificial ha logrado resultados importantes son la cirugía urológica y el diagnóstico de lesiones dérmicas. Recientemente, en el año 2018, la FDA aprobó el uso de un dispositivo oftalmológico que utiliza inteligencia artificial para identificar pacientes con posible retinopatía diabética.

Las aplicaciones de la inteligencia artificial en otorrinolaringología se han centrado en la optimización del diagnóstico genético y proteómico de pacientes con patología oncológica de cabeza y cuello y en el desarrollo de algoritmos para mejorar la detección y comprensión del lenguaje. Es indudable que en el futuro aparecerán nuevas aplicaciones de la inteligencia artificial en nuestra disciplina. Por esta razón, es importante interiorizarse desde ya con los conceptos básicos del aprendizaje de máquinas, y no temer a su desarrollo, pues con seguridad contribuirá a mejorar la calidad de vida de nuestros pacientes.

\section{Dr. Paul Délano R. Director}

Revista de Otorrinolaringología y Cirugía de Cabeza y Cuello 